\title{
Cross-section calculations for positron scattering from pyrimidine over an energy range from 0.1 to $10000 \mathrm{eV}$
}

\author{
A. G. Sanz, ${ }^{1}$ M. C. Fuss, ${ }^{1}$ F. Blanco, ${ }^{2}$ Z. Mašín, ${ }^{3}$ J. D. Gorfinkiel, ${ }^{3}$ R. P. McEachran, ${ }^{4}$ M. J. Brunger,,${ }^{5,6}$ and G. García ${ }^{1,7, *}$ \\ ${ }^{1}$ Instituto de Física Fundamental, Consejo Superior de Investigaciones Científicas, Serrano 113-bis, 28006 Madrid, Spain \\ ${ }^{2}$ Departamento de Física Atómica, Molecular y Nuclear, Universidad Complutense de Madrid, Ciudad Universitaria, 28040 Madrid, Spain \\ ${ }^{3}$ Department of Physical Sciences, The Open University, Walton Hall, Milton Keynes, MK7 6AA, United Kingdom \\ ${ }^{4}$ ARC Centre for Antimatter-Matter Studies, Australian National University, Canberra, ACT 0200, Australia \\ ${ }^{5}$ ARC Centre for Antimatter-Matter Studies, School of Chemical and Physical Sciences, Flinders University, \\ GPO Box 2100, Adelaide, SA 5001, Australia \\ ${ }^{6}$ Institute of Mathematical Sciences, University of Malaya, 50603 Kuala Lumpur, Malaysia \\ ${ }^{7}$ Centre for Medical Radiation Physics, University of Wollongong, NSW 2522, Australia
}

(Received 19 April 2013; published 4 December 2013)

\begin{abstract}
We report a computational investigation of positron scattering by pyrimidine $\left(\mathrm{C}_{4} \mathrm{H}_{4} \mathrm{~N}_{2}\right)$ in the gas phase. Integral and differential cross sections have been calculated over a broad energy range by employing two distinct $a b$ initio quantum scattering methods: the $R$-matrix method and a corrected form of the independent-atom representation, at low and high energies, respectively. Since pyrimidine is a strong polar molecule further dipole-induced excitations have been calculated in the framework of the first Born approximation. Good agreement is found between the different computational models and fair agreement is found with prior experimental results.
\end{abstract}

DOI: 10.1103/PhysRevA.88.062704

\section{INTRODUCTION}

In recent years, positron scattering with molecular targets has attracted great interest in a broad variety of fields of study. These include atomic, molecular, and atmospheric physics as well as the medical science community. New trends in medical procedures, such as positron emission tomography (PET), demand a deeper understanding of the radiation-induced damage of biological systems at the molecular level. Useful tools for modeling this radiation damage include single-track structure simulation software, such as GEANT4 [1], PENELOPE [2], or LEPTS [3], which require as input the interaction probabilities (cross sections) for all the possible scattering processes. In order for these simulations to contribute to good therapeutic outcomes, cross-section data over a very wide energy range are needed.

The electron-molecule dynamics involved in radiation damage is well documented and an intensive effort has been made to include all the electron scattering processes into the simulations, from high energies down to the very low energies of thermalized secondary electrons [4,5]. However studies regarding positron scattering are scarcer and the majority of them have been restricted to a limited energy domain, either at low [6] or high energies [7]. Moreover, the computational analysis of positron scattering with polyatomic molecules is still fairly limited to relatively simple molecular structures [7-9]. In order to extend our knowledge of radiationinduced damage in biomolecular systems and provide relevant parameters for radiation-based biomedical applications, it is necessary to apply these models to increasingly more complicated biomolecules. It is important to note that the great majority of the molecules of biological interest have a considerably high permanent dipole moment, for example, $\mathrm{H}_{2} \mathrm{O}$ [10], the DNA and RNA bases [11-13], tetrahydrofuran

*g.garcia@iff.csic.es
PACS number(s): 34.80.Bm, 34.10.+x, 34.80.Gs

(THF) [14], and the target molecule of the present study pyrimidine [15].

Pyrimidine $\left(\mathrm{C}_{4} \mathrm{H}_{4} \mathrm{~N}_{2}\right)$ is a heterocyclic aromatic organic molecule which contains two nitrogen atoms at positions 1 and 3 of the six-member ring. It has a high permanent dipole moment of $2.334 \mathrm{D}$ [15] and a significant spherical polarizibility of around $60 a_{o}^{3}$ [16]. Pyrimidine is of interest in many fields of study since three of the DNA and RNA bases, namely cytosine, thymine, and uracil, are pyrimidine derivatives. Indeed pyrimidine is normally used as a model compound to investigate, both theoretically and experimentally, radiation scattering from DNA bases. Hence, we have chosen pyrimidine as a benchmark system for testing our quantum positron scattering procedures on multielectron polar targets.

It is well known that significant difficulties arise when attempting to measure experimental cross sections for electron collisions with polar molecules. The main reason for this is that the angular distribution of the scattered charged particles is strongly peaked in the forward direction, but the angular resolution of the experimental apparatus is normally not good enough to distinguish the scattered particles from the primary beam since they are deflected within the finite width of the detector apertures. Furthermore, the excitation energy of the rotational levels is so small that the energy resolution of the experiments is not sufficient to distinguish these inelastic processes from the elastic events. In the case of positron scattering, the experiments are also expected to encounter similar difficulties, plus some additional problems due to the significantly reduced incident positron fluxes. This is reflected in the available data for pyrimidine found in the literature: whereas electron collisions have been the subject of numerous studies, including the measurement of elastic differential cross sections [17,18], gas-phase electronic-state cross-section studies [19,20] and $(e, 2 e)$ ionization studies [21], vibrational and electronic excitation cross sections in the condensed phase [22], and electron energy-loss spectra [23], 
little work on positron scattering from pyrimidine has been done. However, Zecca et al. [24] measured total cross sections (TCSs) from 0.3 to $45 \mathrm{eV}$ using a positron transmission beam technique.

Despite the fact that no exchange effects are present, positron collisions are harder to model than the equivalent scattering of electrons. This is mainly due to the occurrence of positronium formation and the strong correlation-polarization interaction. Furthermore, theoretical studies also suffer from difficulties when modeling the $e^{-}$and $e^{+}$scattering from polar systems since the long-range nature of the dipole potential implies that a large number of partial waves should be included in the wave function expansion. This in turn normally increases significantly the computational requirements. Several studies have provided calculated integral and differential cross sections for electron collisions with pyrimidine at low $[18,25]$ and higher energies $[17,26]$. In addition, we have very recently provided a complete set of recommended integral cross sections calculated over a very broad energy range (1-10000 eV) [27]. However, unfortunately, no theoretical data for positron scattering currently appear to be available in the literature.

The purpose of the present work is therefore to shed some light on positron scattering with a complex polar biomolecule, pyrimidine, in the gas phase. As the above summary suggests, studies of positron collisions have been restricted to relatively simple molecules over a limited energy domain. In the present work, we propose a computational approach, based on the scheme developed for electron scattering in Sanz et al. [4], which combines different quantum scattering models. Accordingly, we employ the $R$-matrix [28] method at low energies, and the corrected form of the independent-atom model [IAM-SCAR (screening-corrected additivity rule)] [29,30] for intermediate to high energies. The $R$-matrix approach is an accurate method to study positron scattering below the positronium formation threshold, as has been shown in Baluja et al. [31] for $e^{+}$-water collisions. The IAM-SCAR method, based on optical potential calculations assuming a modified independent-atom description of the molecular target, constitutes a simple and powerful tool at intermediate and high energies (30-10000 eV) [29,30] which has been successfully applied to electron scattering from a great variety of different-sized target molecules $[4,5,30]$ and to positron scattering from the oxygen molecule [7]. Those references illustrate well the flexibility and potential of this method. This is the same procedure that we successfully applied for electron scattering from pyrimidine [27], with very good numerical agreement between both models at intermediate energies being found, thus confirming the reliability of both methods. Hence, these treatments are expected to provide a suitable computational framework for positron scattering problems with multielectron polar targets including a broad variety of scattering channels. In particular, we provide integral elastic, electronically inelastic, and total cross sections (TCSs), together with elastic differential cross sections (DCSs), for positron scattering from pyrimidine over a broad energy range. Note, however, that in the positron case there is a broader energy range in which neither method is expected to be accurate: our data should be taken to be merely indicative in the $3-30-\mathrm{eV}$ energy range.
In the following section we briefly describe the details of the computational scattering methods we have employed, i.e., the $R$-matrix and IAM-SCAR approaches. In Sec. III we present the details of our calculations and show our integral and differential cross sections at various collision energies. Comparison with the experimental TCSs from Zecca et al. [24] is also made in this section. Finally our work is summarized in Sec. IV, before drawing some conclusions from this study.

\section{THEORETICAL METHODS: AN OUTLINE}

\section{A. $R$-matrix method}

The ab initio molecular quantum mechanical procedure we have employed at low incident energies, the $R$-matrix method, has been discussed in detail in numerous previous works [28], [32,33]. The $R$-matrix theory was first adapted to positron scattering by Tennyson [34] and Danby and Tennyson [35] for diatomic targets. More recently, Baluja et al. [31] extended the $R$-matrix code to polyatomic targets for positron collisions for energies below the positronium (Ps) formation. A few targets have been studied since then $[6,9,36]$. Only a brief summary of the method is therefore given in the present work.

The $R$-matrix method is based on dividing the coordinate space of the particle-molecule collision problem into two regions: an inner region, defined by a sphere typically of radius $a=10-15 a_{\mathrm{o}}$ centered at the center of mass of the molecule, and an outer region. An indispensable condition is that the sphere is sufficiently large to enclose the electronic density of the target states included in the calculation. In the inner region, the complicated many-particle short-range interactions between the scattering particle and the $N$-bound target electrons, i.e., correlation and polarization effects, have to be taken into account. In contrast to the electron-molecule case, the antisymmetrization requirements that lead to exchange do not apply [32] to positrons, so the inner region wave functions are simpler than those for electron scattering. Within these considerations the inner region wave function is therefore expressed by the close-coupling (CC) expansion:

$$
\begin{aligned}
\psi_{k}^{N+1}= & \sum_{i, j} a_{i j k} \phi_{i}\left(x_{1} \cdots x_{N}\right) \tilde{u}_{i j}\left(x_{N+1}\right) \\
& +\sum_{i} b_{i k} \chi_{i}^{N}\left(x_{1} \cdots x_{N}\right) \tilde{\chi}_{i}^{N}\left(x_{N+1}\right),
\end{aligned}
$$

where $k$ represents the $k^{\text {th }}$ solution of the $N$-electron target + positron Hamiltonian in the inner region, $\tilde{u}_{i j}$ are the continuum orbitals describing the scattering positron built from Gaussian-type orbitals (GTOs) centered on the center of mass of the molecule, and $x_{i}$ are the spatial and spin coordinates of electron $i$. In addition, $a_{i j k}$ and $b_{i k}$ are variational coefficients and $\phi_{i}$ are the target wave functions obtained by diagonalizing the Hamiltonian for the $N$-electron target molecule. The terms in the second summation are known as $L^{2}$ functions and are built as products of two functions: $\tilde{\chi}$ denotes a square-integrable spin orbital occupied by the positron, while $\chi_{i}$ is a $N$-electron function. The latter are built as products of target molecular orbitals (occupied and virtual). The $L^{2}$ functions are crucial for the representation of the short-range polarization and correlation effects; they also need to be fully contained inside the $R$-matrix sphere. 
Note that the wave functions of Eq. (1) do not depend on the kinetic energy of the scattering positron and are therefore calculated only once, a feature that provides a substantial advantage over other $a b$ initio methods in terms of computational requirements. In the outer region $(r>a)$, since the correlation effects are negligible, the positrontarget interaction is represented by a long-range multipole expansion. The $R$ matrix is built at the boundary between the regions, using inner region information, and the oneparticle multichannel problem is solved by propagating the $R$ matrix outwards up to a radius large enough so that an asymptotic expansion for the radial wave functions can be matched to known analytical solutions. The corresponding $K$ matrices containing the scattering information are determined, and subsequently integral and differential cross sections are produced via the generation of the necessary $T$ matrices.

The $R$-matrix calculations have been performed at different levels of approximation using the UKRmol suite [37]. The simplest scattering model that we have employed is the static plus polarization (SP) model (in analogy with the static-exchange plus polarization (SEP) for electronmolecule scattering). In this model only the ground state of the molecule is considered; its wave function is described at the Hartree-Fock level. The molecule is allowed to be polarized by the incoming positron through the $L^{2}$ configurations: one electron from the valence space of the target is promoted to one of a selected number of virtual orbitals (i.e., orbitals that are not occupied in the ground-state configuration). Because the Pauli exclusion principle does not apply to a positron-electron pair, the scattered positron can occupy any of the target molecular orbitals, either the virtual orbitals (i.e., unoccupied) or those already doubly occupied by electrons. These configurations are only constrained to satisfy both the electronic-spin and overall space-spin symmetry [38].

Additionally, we have used the close-coupling (CC) approximation in which, apart from the ground state, a number of excited target electronic states are included in the CC expansion. Hence, integral electronically inelastic cross sections can be obtained. The target wave functions are calculated at the complete active space self-consistent field (CASSCF) level. In the present calculations we included the 14 lowest singlet excited states. No ionized states were considered as this would have required the inclusion of pseudostates and would have therefore increased drastically the computational requirements. Moreover the Ps formation channel is not described within our $R$-matrix implementation. This means that above the Ps formation threshold, which for pyrimidine lies at $2.93 \mathrm{eV}$ [24], the computed cross sections are less accurate than those for electrons. Nevertheless, cross sections were computed with the $R$-matrix procedure up to $20 \mathrm{eV}$, in order to compare with the available experimental TCS data and the theoretical IAM-SCAR cross sections at intermediate energies.

It should be noted that the $R$-matrix method treats the scattering problem within the fixed nuclei approximation, which considers the time scale of the collision to be short compared to the nuclear motions [39]. In other words, vibrations and rotations are assumed to be slow compared to the velocity of the incident positron. Hence, the nuclei are treated as fixed particles during the collision process: the equilibrium geometry of the molecule is unaltered. Although this approximation simplifies significantly the scattering equations and gives reliable results for nonpolar targets, it is known to fail for molecules with a high permanent dipole moment. This is because the cross sections diverge due to the long-range nature of the positron (electron)-dipole interaction, leading to divergences in the elastic DCS mainly in the forward direction [40]. A widespread procedure to avoid these undesirable divergences is based on using the first Born approximation (FBA) for a charged particle in a point-dipole potential [41-43], since the contributions from all the partial waves, both individually and as summed quantities [44], can be calculated analytically within the FBA. Based on this approximation a variety of Born "top-up" procedures [45,46] have been developed in the past [6]. Among them we have chosen the frame-transformation method, implemented by Sanna and Gianturco [45] in the POLYDCS code, which is in turn based on the multipole-extracted adiabatic-nuclei (MEAN) procedure proposed by Norcross and Padial [42]. Within this approach, after applying a frame-transformation scheme from the body-fixed to the space-fixed frame of reference in order to allow for rotational motion, slow convergence of the partial-wave expansion can be avoided by using the following expression:

$$
\frac{d \sigma}{d \Omega}=\frac{d \sigma^{B}}{d \Omega}+\sum_{L}^{2 l_{\max }+1}\left(A_{L}-A_{L}^{B}\right) P_{L}[\cos (\theta)] .
$$

Note that $d \sigma / d \Omega$ is now obtained for an initial rotational state as a sum over final rotational states. The contribution to the DCS from low-partial waves (up to $l_{\max }$ ) is calculated $a b$ initio, in this work with the UKRmol suite, so that shortrange effects are considered. In this way, collisions leading to (dipole-forbidden) transitions with $\Delta j \neq 1$, which are known to be dominated by low-partial waves [47], are also taken into account. The higher partial waves are introduced by calculating the cross sections using the Born approximation and then subtracting the partial cross sections for the low-partial waves already described by the ab initio data. Hence, the formula given by Eq. (2) can be understood as a short-range correction to the original Born approximation [6]. The present cross sections were calculated assuming the molecule is a symmetric top. We have only considered transitions from the ground state $(j=0)$ of pyrimidine to rotational states up to $j=9$ (and all possible $\tau$ values for each $j$ ). Since the initial population of rotational states is not taken into account, these results should be seen as $0 \mathrm{~K}$ cross sections. Although still an approximate approach, the Born correction implemented in POLYDCs has been shown to produce consistent rotationally summed integral and differential cross sections for various biomolecules of arbitrary geometry $[4,6,25,27]$.

\section{B. Screening-corrected additivity rule (SCAR) method}

At intermediate and high energies we apply the wellknown corrected form of the independent-atom model (IAM), the so-called SCAR (screening-corrected additivity rule) approach. Specific details for this procedure, as applied to electron-molecule scattering, have been extensively described in previous works $[29,30,48,49]$. This method has also been 
recently adapted to positron scattering from argon [50] and molecular oxygen [7], finding reasonable agreement with the reported experimental values. In those latter works an exhaustive description of the changes introduced for positron scattering is given. Hence, we only briefly summarize here the method we used in the present calculations.

In contrast to the aforementioned low-energy $R$-matrix method, IAM-SCAR does not consider the molecule as a single target but, instead, substitutes it by its constituent atoms in their corresponding molecular positions by assuming that the molecular binding does not affect the electronic distribution of the atoms. Each atom is supposed, therefore, to scatter independently. One of the great advantages of this approach is the possibility of obtaining cross sections for a large number of molecular species from a reduced number of atoms. Consequently, the initial concern of these calculations is the interaction probabilities for the constituent atoms of the pyrimidine molecule, specifically: H, C, and N. Each atomic target is represented by an interacting complex optical potential, $V_{\text {opt }}(\mathbf{r})$, whose real part represents the positron-atom elastic interactions while the imaginary part accounts for the inelastic collisions considered as absorptions from the incident positron beam. We use

$V_{\text {opt }}(r)=V_{R}(r)+V_{\mathrm{abs}}(r)=V_{s}(r)+V_{\mathrm{pol}}(r)+V_{\mathrm{abs}}(r)$.

The real part of the potential consists of the electrostatic $\left[V_{s}(r)\right]$ and the polarization $\left[V_{\mathrm{pol}}(r)\right]$ terms. The former describes the repulsive static interaction between the incident positron and the atomic charge distribution (electrons + nuclei), which have been derived from Hartree-Fock calculations of the atomic charge density following a procedure similar to the one proposed by Reid and Wadehra [51-53]. The effect of the deformation of the target electronic cloud during the positron collision is given by the polarization potential. Polarization effects normally present as a quadratic dependence on the applied fields, being therefore independent of the charge sign of the projectile. This means that the polarization term could in principle be the same as for electron scattering. However, the choice of the polarization term is, in principle, important in the positron-molecule scattering dynamics as it is the only attractive contribution to the positron-target interaction to counteract the repulsive nature of the static potential since no exchange term exists. Consequently, the computed cross sections are very sensitive to the procedure chosen to model the polarization potential and a careful evaluation is indispensable. Based on the experience gained in previous work [7] with molecular oxygen $\left(\mathrm{O}_{2}\right)$, instead of employing the polarization potential of the form given by Zhang et al. [54], usual for electron scattering [4], we use a polarization potential based on that proposed by McEachran et al. [55] for noble gases. In particular we have employed the dipole $\left(V_{d}\right)$ and the dipole plus quadrupole polarization $\left(V_{d+p}\right)$ potentials for $\mathrm{Ne}$, as described in McEachran et al. [55], but scaled by a constant in order to reproduce the known dipole $\left(\alpha_{d}\right)$ and quadrupole polarizibility ( $\left.\alpha_{q}\right)$ of $\mathrm{H}\left(\alpha_{d}=4.5\right.$ a.u. [56], $\alpha_{q}=15$ a.u. [57]), $\mathrm{N}$ ( $\alpha_{d}=7.63$ a.u. [58], $\alpha_{q}=25.66$ a.u. [59]), and C $\left(\alpha_{d}=11.88\right.$ a.u. [60], $\alpha_{q}=54.76$ a.u. [59]). It has been shown that the inclusion of a dipole plus a quadrupole polarization potential in the calculation results in very good agreement with the experimental data for the positron-neon elastic cross sections [61]; this has therefore encouraged us to consider this potential in our study (for further details see Chiari et al. [7]). It should be noted that the nonadiabatic polarization terms were not included in the present calculations. The importance of including dynamic distortion effects has been shown by Mimnagh et al. [62] for electrons and it will be the subject of further investigation in order to improve the accuracy of our scattering potential both for electrons and positrons.

The absorption potential is derived following the scheme developed by Reid and Wadehra [51-53], which is in turn based on the procedure proposed by Staszewska et al. [63] for electron scattering. The inelastic scattering is treated as binary collisions between the incident particle and the target electrons represented as a quasifree electron cloud. In the case of electron scattering, some improvements to the original form were included leading to a model of reasonable simplicity and accuracy over a wide energy range, while maintaining the $a b$ initio nature. The most controversial point of this procedure is the definition of the energy for the absorption threshold $(\Delta)$. For electron scattering it is customary to take $\Delta$ as the excitation energy of the first electronic state. However, for positron collisions an additional scattering channel exists: positronium (Ps) formation, whose threshold, $\Delta_{p}$, is at $6.8 \mathrm{eV}$ below the ionization potential:

$$
\Delta_{p}=V_{i}-6.8 \mathrm{eV}
$$

The ionization potential for our present target molecule, pyrimidine, is at $9.7 \mathrm{eV} \mathrm{[64]} \mathrm{placing} \mathrm{the} \mathrm{positronium} \mathrm{formation}$ threshold at $2.93 \mathrm{eV}$ [24], using Eq. (4). Note that the Ps formation threshold lies below the first electronic-state excitation energy, where it constitutes the dominant inelastic scattering channel. Since Ps formation cannot be described in terms of a binary collision [65], it cannot be explicitly introduced into the original formulation of the absorption potential as an independent inelastic event. In an earlier attempt to deal with this problem, Reid and Wadehra [52] proposed to define the threshold absorption parameter as the Ps formation energy, i.e., $\Delta=\Delta_{p}$, lowering therefore the energy to initiate the absorption processes. It was later shown that the main drawback of this approach is that the total cross sections are overestimated for energies above $100 \mathrm{eV}$ [7]. The solution that we adopt is therefore to define an energy-dependent parameter for the absorption threshold:

$$
\Delta(E)=\Delta_{e}-\left(\Delta_{e}-\Delta_{p}\right) e^{-\left(\frac{E-\Delta_{p}}{E_{m}}\right)},
$$

where $\Delta_{e}$ is the lowest excitation energy of the atomic targets, $\Delta_{p}$ is the Ps formation threshold, and $E_{m}$ is a characteristic energy at which the inelastic cross section, without positronium formation, reaches its maximum $\left(E_{m}=\right.$ $20 \mathrm{eV}$ in this case). This expression provides values between the limit conditions: $\Delta(E)=\Delta_{p}$ for energies close to the Ps formation threshold and $\Delta(E)=\Delta_{e}$ for higher energies. A smooth transition between both limits is modulated by the negative exponential and governed by the $E_{m}$ parameter. Note that $E_{m}$ is not a critical parameter and no appreciable differences are found when the value is varied in a $20 \%-30 \%$ range.

As a second step, molecular cross sections are computed from the atomic data by applying a coherent addition, 
commonly known as the additivity rule (AR) [66,67]. This procedure gives reasonable results for energies above $100 \mathrm{eV}$, as the incident particles are fast enough to effectively "see" the target molecule as a sum of the individual atoms. For lower energies, the atomic cross sections are sufficiently large to overlap, leading to an overestimation of the molecular cross sections. This limitation has been at least partially resolved by introducing some screening coefficients which modify both the differential and integral cross sections, as implemented by Blanco and García [29,30] in the SCAR code. This method has proven to be a powerful tool to calculate cross sections down to about $30 \mathrm{eV}$, at least for electron scattering, as shown in previous work [68-70] for several targets. In a recent study for the oxygen molecule, Chiari et al. [7] also showed the reliability of the present approach for positron scattering.

From the previous description we can infer that both vibrational and rotational excitations are ignored in the IAMSCAR method. However, in the case of polar targets such as pyrimidine, dipole-induced rotational excitations are not negligible and must be included in the scattering calculations. The method we follow in this case, based on the one suggested by Jain [71], assumes the interaction of a charged particle with a free electric dipole in the framework of the first Born approximation. Then the calculated differential and integral rotational excitation cross sections are incorporated within our IAM-SCAR calculation in an incoherent way, i.e., by adding the results as a channel independent from the other channels. Although rotational excitation energies are, in general, fairly small (typically a few $\mathrm{meV}$ ) in comparison with the incident positron energies, in order for the FBA to be valid, the latter energies should be higher than about $2 \mathrm{eV}$. Under these circumstances, rotational excitation cross sections are calculated by weighting the population for the $j$ th rotational quantum level at $300 \mathrm{~K}$ and estimating the average excitation energy from the corresponding rotational constants. The complete approach has been shown to be quite successful when applied to some polar molecules [72,73]. However, when the target molecule has a strong permanent dipole moment, as is the case of many biomolecules like pyrimidine, it is known that the FBA fails for medium and large scattering angles $(\theta)$. In order to partially solve this problem we incorporated a correction based on that suggested by Dickinson [74], which introduces a first-order corrective term to the differential cross sections for medium and large angles but maintains the FBA correction for lower angles so that

$$
\frac{d \sigma^{B}}{d \Omega} \approx \frac{|\mu|^{2}}{6 E} \frac{1}{\sin ^{2}\left(\frac{\theta}{2}\right)} \quad \theta<\theta_{c},
$$

and

$$
\frac{d \sigma^{D c k}}{d \Omega} \approx \frac{\pi|\mu|}{64 E} \frac{1}{\sin ^{3}\left(\frac{\theta}{2}\right)} \quad \theta>\theta_{c},
$$

where $\mu$ is the magnitude of the permanent dipole moment of the molecule and $E$ the energy of the projectile. Provided that the dipole moment is larger than $\mu=0.75 \mathrm{D}$, both curves smoothly join together at a specific angle $\theta_{c}$, i.e., the critical angle where they cross each other. Successful results have been obtained in the past by applying this correction to electron scattering from $\mathrm{HCN}$ [4]. Given that the dipole-induced excitations are independent of the charge sign of the incident particle, we expect to obtain quite accurate data also for positron scattering.

\section{DISCUSSION}

\section{A. Details of the calculations}

All calculations have been performed at the equilibrium geometry of pyrimidine [75], which belongs to the $C_{2 v}$ point group. In the low-energy range, cross sections have been computed with the $R$-matrix method after adapting the models developed for electron scattering [28]. We have used two different basis sets: a compact cc-pVDZ basis, and a diffuse $6-311+\mathrm{G}^{* *}$ basis. In order to guarantee that the electronic density of all the target states included in the calculation is negligible outside the $R$-matrix sphere, we have used a $R$-matrix radius of $a=13 a_{\mathrm{o}}$ and $a=18 a_{\mathrm{o}}$, for the compact and the diffuse basis sets, respectively. The scattered positron is described by the continuum basis set developed by Faure et al. [76] which includes partial waves up to either $l=4$ or $l=5$. Hartree-Fock SCF and state-averaged CASSCF [an active space $(10,8)$ was employed] orbitals were generated using MOLPRO [77], for the SP and CC calculation, respectively. We have included the 14 lowest singlet electronic states of pyrimidine in the close-coupling expansion. As stated by Mašín et al. [25,33], these calculations are very sensitive to the nature of the basis set used. For instance, the virtual orbitals, important for modeling correlation and polarization effects, were found to be considerably different in energy and shape when using the compact or the diffuse basis set. These differences manifest themselves in the number of virtual orbitals used to generate $L^{2}$ functions for the different models: 35-40 for the SP and CC calculation, respectively, for the compact basis set and 40 orbitals for the SP calculations with the diffuse basis set. Pyrimidine possesses a significant permanent dipole moment: 2.334 D [15]. The dipole moment obtained with the compact basis set was 2.31 and 2.36 D for the Hartree-Fock and CASSCF models, respectively; for the diffuse basis set the value obtained was slightly higher: $2.53 \mathrm{D}$. Dipole-corrected cross sections have been calculated using POLYDCS for each model employing the respective computed dipole moment.

Cross sections have been computed by employing the IAMSCAR model for $E>1 \mathrm{eV}$ (but deemed accurate for energies above $20 \mathrm{eV}$ ). The corresponding atomic cross sections for $\mathrm{C}, \mathrm{H}$, and $\mathrm{N}$ have been previously calculated and discussed (Blanco and Garcia [49] and references therein for the case of electrons). For this energy range, the intrinsic estimated numerical uncertainty is about $10 \%$, as discussed by us in previous works [5,72]. We believe a similar level of accuracy is also achieved for positron scattering. The accuracy of the $R$-matrix procedure is harder to gauge. The comparison [25] with experimental results for electron-pyrimidine collisions indicates that the elastic cross sections are in very good agreement if the small-angle scattering $\left(\theta<20^{\circ}\right)$ is not taken into account. Since the Ps formation channels are absent from the calculations, the accuracy of the $R$-matrix cross sections above $\sim 3 \mathrm{eV}$ is inevitably lower than that of electron scattering cross sections for the same target. The Ps formation cross 
TABLE I. Present integral elastic cross sections, both dipole corrected (rotationally summed) and uncorrected for positronpyrimidine scattering from 0.5 to $10 \mathrm{eV}$, calculated with the $R$-matrix approach both at the static-polarization and close-coupling model (with compact basis set cc-pVDZ and $l \leqslant 4$ ).

\begin{tabular}{|c|c|c|c|c|}
\hline \multirow[b]{2}{*}{$\begin{array}{l}\text { Energy } \\
(\mathrm{eV})\end{array}$} & \multicolumn{2}{|c|}{$R$-matrix SP model } & \multicolumn{2}{|c|}{$R$-matrix CC model } \\
\hline & $\left(\AA^{2}\right)$ & $\begin{array}{c}\text { Dipole } \\
\text { corrected }\left(\AA^{2}\right)\end{array}$ & Elastic $\left(\AA^{2}\right)$ & $\begin{array}{c}\text { Dipole } \\
\text { corrected }\left(\AA^{2}\right)\end{array}$ \\
\hline 0.50 & 84.26 & 616.22 & 103.52 & 635.13 \\
\hline 1.00 & 42.62 & 326.71 & 53.13 & 338.35 \\
\hline 1.50 & 29.43 & 225.94 & 38.58 & 235.98 \\
\hline 2.00 & 23.35 & 174.77 & 32.20 & 184.08 \\
\hline 2.50 & 20.02 & 143.46 & 28.64 & 152.30 \\
\hline 3.00 & 17.98 & 122.56 & 26.34 & 131.04 \\
\hline 5.00 & 14.52 & 79.91 & 21.78 & 86.65 \\
\hline 10.00 & 13.38 & 47.85 & 18.18 & 52.61 \\
\hline
\end{tabular}

section peaks around $10 \mathrm{eV}$; its size is about $20 \%$ that of the elastic cross section. This is approximately the additional uncertainty that applies to the elastic and total positron cross sections.

\section{B. Elastic cross sections}

The calculated integral elastic cross sections computed with the $R$-matrix (Table I) in the low-energy regime are shown in Fig. 1(a). Note that several different $R$-matrix models have been used. In particular SP calculations have been performed using the compact and diffuse basis sets (dashed-dotted blue thin line and dashed light-green thin line, respectively) with partial waves in both cases up to $l=4$. The CC calculations were performed using the compact basis set and partial waves up to $l=4$ (solid dark-green thin line). These refer to elastic integral cross sections (ICSs) that are not Born corrected, but the corresponding Born-corrected elastic ICS can also be found in Fig. 1(a). We have confirmed by running tests, with the compact basis set, that increasing the partial waves included in the $a b$ initio calculations up to $l=5$ has no discernible effect on the cross sections. The numerous narrow peaks visible for energies above $\sim 5 \mathrm{eV}$ in the ab initio SP cross sections correspond to (nonphysical) pseudoresonances, an inherent feature of the SP approximation when using a multiconfiguration description for the $(N+1)$-particle wave function. The cross sections obtained with the diffuse basis set are bigger in magnitude than those for the compact basis set. We also observe that the pseudoresonances tend to appear at lower energies in this case. The SP and CC approximations produce very similar results for the compact basis set, especially at low energies, although some slight divergences seem to arise with increasing energies. This behavior was already noticed in our previous electron scattering calculations [25], where cross sections generated with the SEP and CC models agree very well, with the exception of the resonance positions which are better described in the former approximation. It is customary therefore to employ each of these approximations in a limited energy range to optimize the results: at low energies, the SP model is preferred since it provides a better representation of the short-range polarization and correlation effects. For energies above the first singlet excitation threshold, which for pyrimidine is around $4 \mathrm{eV} \mathrm{[78],} \mathrm{the} \mathrm{CC} \mathrm{model} \mathrm{is} \mathrm{preferred} \mathrm{as}$ it accounts for the electronic-state excitations.

Integral elastic cross sections computed with the IAMSCAR method (Table II), at intermediate and high energies, are presented in Fig. 1(b). As mentioned earlier, two different models for the polarization potential were used: $V_{d}$ [solid black line $(\bullet)]$ and $V_{d+p}$ [solid blue line $(\times)$ ]. Although both models show similar qualitative behavior, we find that cross sections generated with the dipole plus quadrupole potential are larger in magnitude. Note again that in this optical model
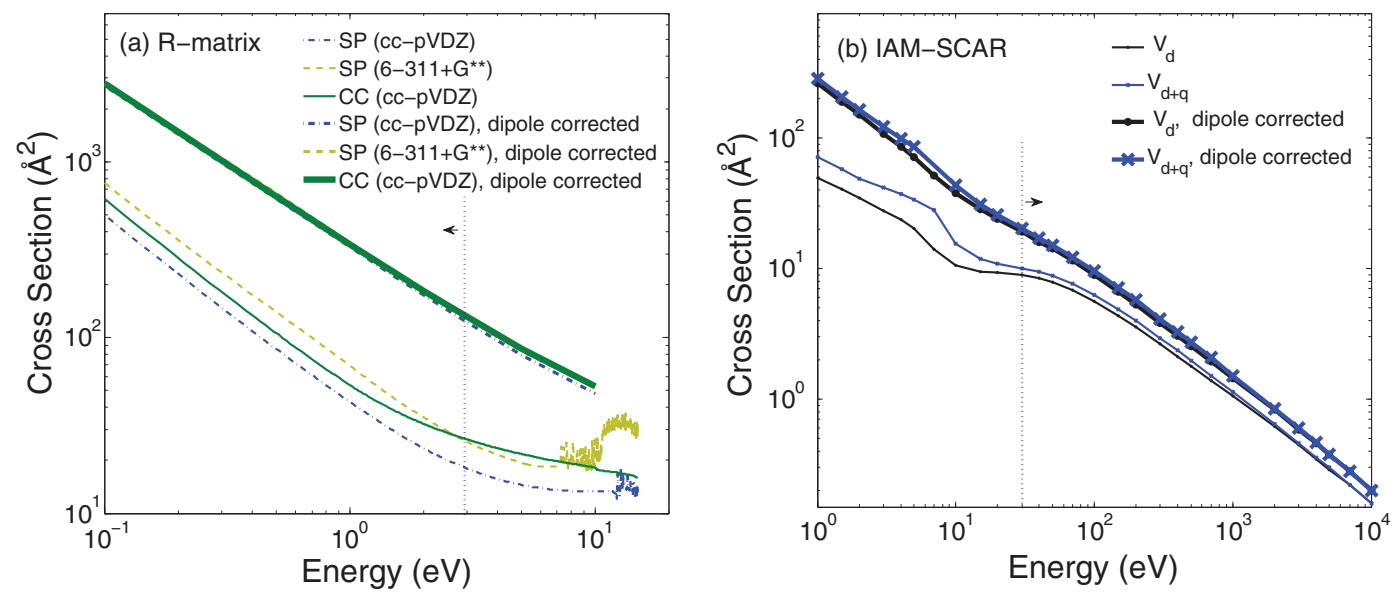

FIG. 1. (Color online) Present integral elastic cross sections for positron scattering by pyrimidine computed with: (a) the $R$-matrix method at the SP level using the cc-pVDZ basis set (dashed-dotted blue line) and the diffuse basis set 6-311+ $\mathrm{G}^{* *}$ (dashed light-green line), and at the CC level (cc-pVDZ) (solid dark-green line). Both uncorrected (thin lines) and Born-corrected (thick lines) cross sections are given. This quantum scattering method is expected to be valid up to the Ps formation threshold, which is indicated by a black-dotted line. (b) The IAM-SCAR method with the dipole polarization potential [solid black thin line $(\bullet)$ ] and dipole plus quadrupole polarization potential [solid blue thin line $(x)$ ], also uncorrected (thin lines) and including the Born dipole allowed rotational excitations (thick lines). The region of validity of this method, namely for energies above about $30 \mathrm{eV}$, is also indicated by a black-dotted line, which is placed at $30 \mathrm{eV}$ (see text for details). 
TABLE II. Present integral cross sections for elastic, inelastic (rotational excitations, electronic-state excitations, positronium formation, and ionization) and total scattering for positron-pyrimidine collisions from 1 to $10000 \mathrm{eV}$, as calculated with the IAM-SCAR approach both with the dipole polarization potential and dipole plus quadrupole potential.

\begin{tabular}{|c|c|c|c|c|c|c|}
\hline \multirow[b]{2}{*}{ Energy $(\mathrm{eV})$} & \multicolumn{3}{|c|}{ IAM-SCAR with dipole potential } & \multicolumn{3}{|c|}{ IAM-SCAR with dipole plus quadrupole potential } \\
\hline & Elastic $\left(\AA^{2}\right)$ & Inelastic $\left(\AA^{2}\right)$ & Total $\left(\AA^{2}\right)$ & Elastic $\left(\AA^{2}\right)$ & Inelastic $\left(\AA^{2}\right)$ & Total $\left(\AA^{2}\right)$ \\
\hline 1.0 & 49.56 & 212.52 & 262.08 & 71.12 & 212.80 & 283.92 \\
\hline 1.5 & 40.32 & 148.96 & 189.28 & 57.40 & 148.96 & 206.36 \\
\hline 2 & 34.72 & 115.36 & 150.08 & 48.72 & 115.36 & 164.08 \\
\hline 3 & 27.78 & 80.36 & 108.36 & 41.72 & 80.36 & 122.08 \\
\hline 4 & 23.60 & 62.16 & 85.96 & 37.24 & 62.16 & 99.40 \\
\hline 5 & 20.38 & 52.08 & 72.24 & 33.88 & 53.02 & 86.90 \\
\hline 7 & 14.08 & 48.72 & 62.72 & 28.00 & 58.49 & 86.49 \\
\hline 10 & 10.53 & 47.6 & 57.96 & 15.46 & 47.60 & 63.06 \\
\hline 15 & 9.44 & 43.4 & 52.64 & 11.82 & 43.40 & 55.22 \\
\hline 20 & 9.30 & 39.48 & 48.72 & 10.92 & 39.48 & 50.40 \\
\hline 30 & 8.96 & 33.88 & 42.84 & 10.08 & 33.88 & 43.96 \\
\hline 40 & 8.46 & 29.96 & 38.64 & 9.41 & 29.96 & 39.37 \\
\hline 50 & 7.92 & 27.412 & 35.28 & 8.76 & 27.41 & 36.18 \\
\hline 70 & 6.86 & 23.744 & 30.52 & 7.62 & 23.74 & 31.36 \\
\hline 100 & 5.63 & 20.216 & 25.844 & 6.30 & 20.22 & 26.52 \\
\hline 150 & 4.34 & 16.52 & 20.86 & 4.87 & 16.52 & 21.39 \\
\hline 200 & 3.56 & 14.084 & 17.64 & 3.98 & 14.08 & 18.06 \\
\hline 300 & 2.64 & 11.004 & 13.664 & 2.94 & 11.00 & 13.94 \\
\hline 400 & 2.13 & 9.1 & 11.228 & 2.36 & 9.10 & 11.46 \\
\hline 500 & 1.80 & 7.812 & 9.604 & 1.98 & 7.81 & 9.79 \\
\hline 700 & 1.39 & 6.104 & 7.504 & 1.52 & 6.10 & 7.62 \\
\hline 1000 & 1.06 & 4.62 & 5.684 & 1.14 & 4.62 & 5.76 \\
\hline 2000 & 0.62 & 2.632 & 3.248 & 0.65 & 2.63 & 3.28 \\
\hline 3000 & 0.45 & 1.862 & 2.31 & 0.46 & 1.86 & 2.32 \\
\hline 4000 & 0.35 & 1.4476 & 1.8004 & 0.36 & 1.45 & 1.81 \\
\hline 5000 & 0.29 & 1.1872 & 1.4812 & 0.30 & 1.19 & 1.49 \\
\hline 7000 & 0.22 & 0.8764 & 1.0976 & 0.22 & 0.88 & 1.10 \\
\hline 10000 & 0.16 & 0.63 & 0.7896 & 0.16 & 0.63 & 0.79 \\
\hline
\end{tabular}

calculation the polarization potential is the only attractive interaction between the positron and the target. Consequently, the attractive interaction is enhanced when the quadrupole term is included, leading to larger cross sections in particular at low energies. However, at higher energies the results from both methods converge.

The elastic ICSs from the different $R$-matrix and IAMSCAR models are presented together in Fig. 2. They are in pretty good agreement with one another, to within their respective ranges of validity. However, we clearly discern in this figure some discrepancies between the uncorrected cross sections for both methods at intermediate energies. The origin of these discrepancies may be due to the different methods for treatment of the polarization effects, since the results are very sensitive to the description of the polarization potential. However, once the cross sections are Born corrected (thick lines in Fig. 2), allowing therefore for rotational dipole allowed transitions, the agreement between the $R$-matrix and IAM-SCAR results is now markedly improved. This is because positron scattering from polar molecules is dominated by the dipole interaction, correlation and polarization being weaker effects. Under these circumstances, cross sections from the polar target pyrimidine show therefore very little dependence on the method used and thus on the treatment employed to model the polarization interaction, as observed for water [31].

The angular distributions for the elastic scattering process are shown in Fig. 3, where we report DCS calculated with the $R$-matrix-POLYDCS and IAM-SCAR (dipole-corrected) methods for some selected incident positron energy values. In accordance with the integral cross sections, $R$-matrix results from the SP model are larger in magnitude when using the diffuse basis set (dashed-green line) than when the cc-pVDZ basis is employed (dashed-dotted blue line), although the energy dependence of the DCS generated is similar for both basis sets. The DCSs computed with the CC model are slightly larger in magnitude than the SP results, in particular within the $20^{\circ}-60^{\circ}$ angular range. The IAM-SCAR approach results using the dipole plus quadrupole [solid blue line $(x)$ ] polarization potential generates larger cross sections than when the dipole polarization potential alone is employed [solid black line $(x)$ ], for the angular region above $40^{\circ}$ and at low energies. However, with increasing energies, both these approximations tend to converge in their DCSs and from $50 \mathrm{eV}$ they are almost equal. As already discussed the Born approximation is known to fail from intermediate angles up to $180^{\circ}$, whenever the permanent dipole moment of the target molecule is very large. We have shown in previous works that this failure is partially 


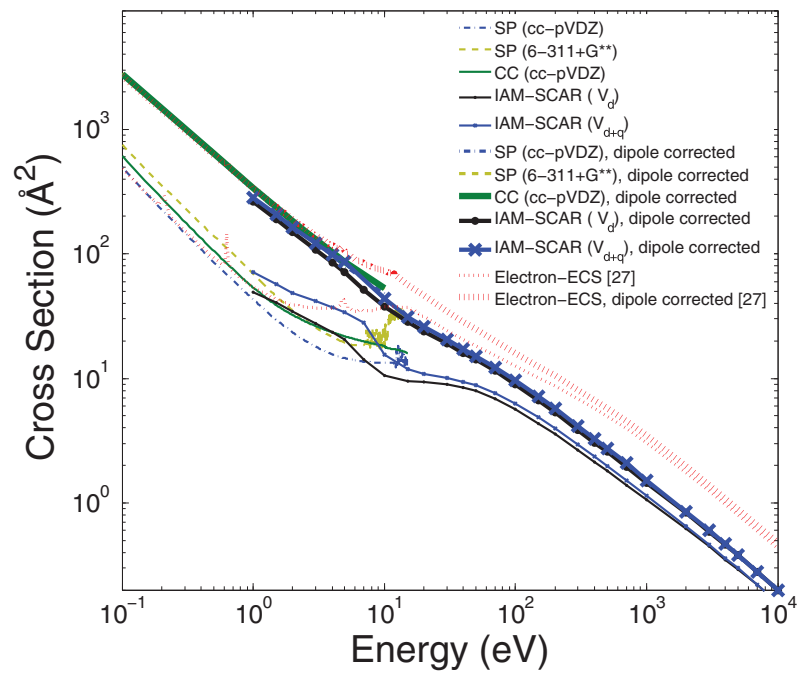

FIG. 2. (Color online) The present computed integral elastic cross sections for positron scattering by pyrimidine computed with the $R$-matrix method (at the SP and CC levels of approximation) and with the IAM-SCAR method (using $V_{d}$ and $V_{d+p}$ ), with and without Born-type correction (same legend as in Fig. 1). Additionally we have plotted, for comparison, the electron scattering elastic integral cross sections with (dotted red thick line) and without (dotted red thin line) the Born correction, as obtained by combining the $R$-matrix [25] and IAM-SCAR methods as shown in Sanz et al. [27].

addressed when the Dickinson correction is applied to the IAM-SCAR approach (dashed-dotted blue line), providing a more realistic representation at medium and large scattering angles (for further details see Sanz et al. $[4,27]$ ). The angular dependence of the $R$-matrix DCS is characterized by a shoulder at around $40^{\circ}-60^{\circ}$, which is progressively shifted to lower angles as the impact energy increases (i.e., it is at around $20^{\circ}$ for $10 \mathrm{eV}$ ). In contrast the IAM-SCAR angular distribution shows a broad minimum at around $90^{\circ}-110^{\circ}$ for low energies, which is fairly pronounced at $10 \mathrm{eV}$, but tends to disappear at higher energies. We also observe that all our calculated curves, converging in value for angles below $20^{\circ}$, are strongly peaked in the forward direction, as expected due to the strong polar nature of pyrimidine. This behavior is in fact more dramatic as the incident positron energy decreases. Where a comparison is possible between the results from both methods, i.e., below $\sim 10 \mathrm{eV}$ [Figs. 3(a)-3(d)], although in general there is reasonable qualitative agreement, we see that discrepancies arise for angles above $20^{\circ}$, which we attribute to the limitation of the IAM-SCAR method in this low-energy range. Note, also, that the $R$-matrix results at $10 \mathrm{eV}$ and above are merely indicative, as both the positronium formation and ionization channels are missing from those calculations.

Unfortunately, there are no experimental or other theoretical elastic positron cross sections available in the literature, to compare with our present data. Therefore, we have to rely on the agreement achieved by both our $R$-matrix and IAMSCAR models when applied to electron-pyrimidine collisions to ascertain the validity of our results. We have recently shown [25] that very good agreement is attained between the $R$-matrix-POLYDCS differential cross sections and the experimental data provided by Palihawadana et al. [18] for electron energies up to $15 \mathrm{eV}$. The IAM-SCAR theory also agrees well with the measurements from the Belgrade group [17] above $50^{\circ}$, although it fails to reproduce the characteristic shoulder that also appears for electron-pyrimidine collisions around $40^{\circ}$. However, as the energy further increases, the independent-atom model comes into better agreement throughout the whole angular range with the experimental data. In addition, very good numerical agreement was found between both methods at intermediate energies, confirming therefore their consistency for the elastic electron scattering process. Our recommended theoretical $e^{-}$-pyrimidine elastic integral cross sections, uncorrected (dotted red thin line) and the corresponding Born-corrected (dotted red thick line), and the elastic differential (Born-corrected) cross sections (dotted red line), obtained by means of combining the results from the $R$-matrix and IAM-SCAR methods [27], are shown in Figs. 1 and 2, respectively. In order to provide a coherent comparison with the positron data, the electron scattering dipole-corrected cross sections have been recalculated extending the rotational transitions up to $j=9$. We have found that for the diazine molecules (and 1,4-dioxane), the inclusion of the rotational transitions up to $j=9$ is essential for the convergence of the cross sections. In addition, for energies above the first excitation threshold, we have employed the $T$ matrix of the CC calculation (instead of the $K$ matrix) and a slightly modified POLYDCS code, since it leads to more accurate dipole-corrected cross sections [79]; this has been done for both the positron and electron cross sections. When comparing the positron and electron scattering cross sections, the most obvious difference is that no shape resonances are present when the incident particle is a positron (see Fig. 2). With this exception, the electron and positron elastic DCS are similar, although the positron cross sections tend to be lower in magnitude. This decrease in size is not unexpected: firstly, no exchange interaction exists when the incident particle is a positron. In addition, whereas both the static and the polarization interaction are attractive for electrons, the static interaction becomes repulsive for positrons. Consequently, the elastic scattering cross sections for incident positrons are lower in magnitude than those for electron scattering. We also note that both the electron- and positron-impact cross sections are strongly peaked in the forward direction, confirming therefore that the dipole interaction dominates over the static interaction, in particular at low energies, independent of the sign of the incident particle charge (see Figs. 2 and 3).

\section{Inelastic and total cross sections}

Theoretical inelastic and total cross sections are plotted in Fig. 4. In the low-energy domain, the cross sections are computed with the $R$-matrix method at the close-coupling level and including 14 excited states. As mentioned above, since the $R$-matrix calculation includes neither the Ps formation description, nor a description of ionization, the inelastic ICS calculated with this method (dashed-dotted blue thick line) is merely indicative and accounts only for the electronic excitations. A Born correction has also been added to the inelastic ICS, in order to account for the higher partial waves (i.e., $l>4$ ) not included in the ab initio calculation [80]. Note that the corrected cross sections give a better representation for the 

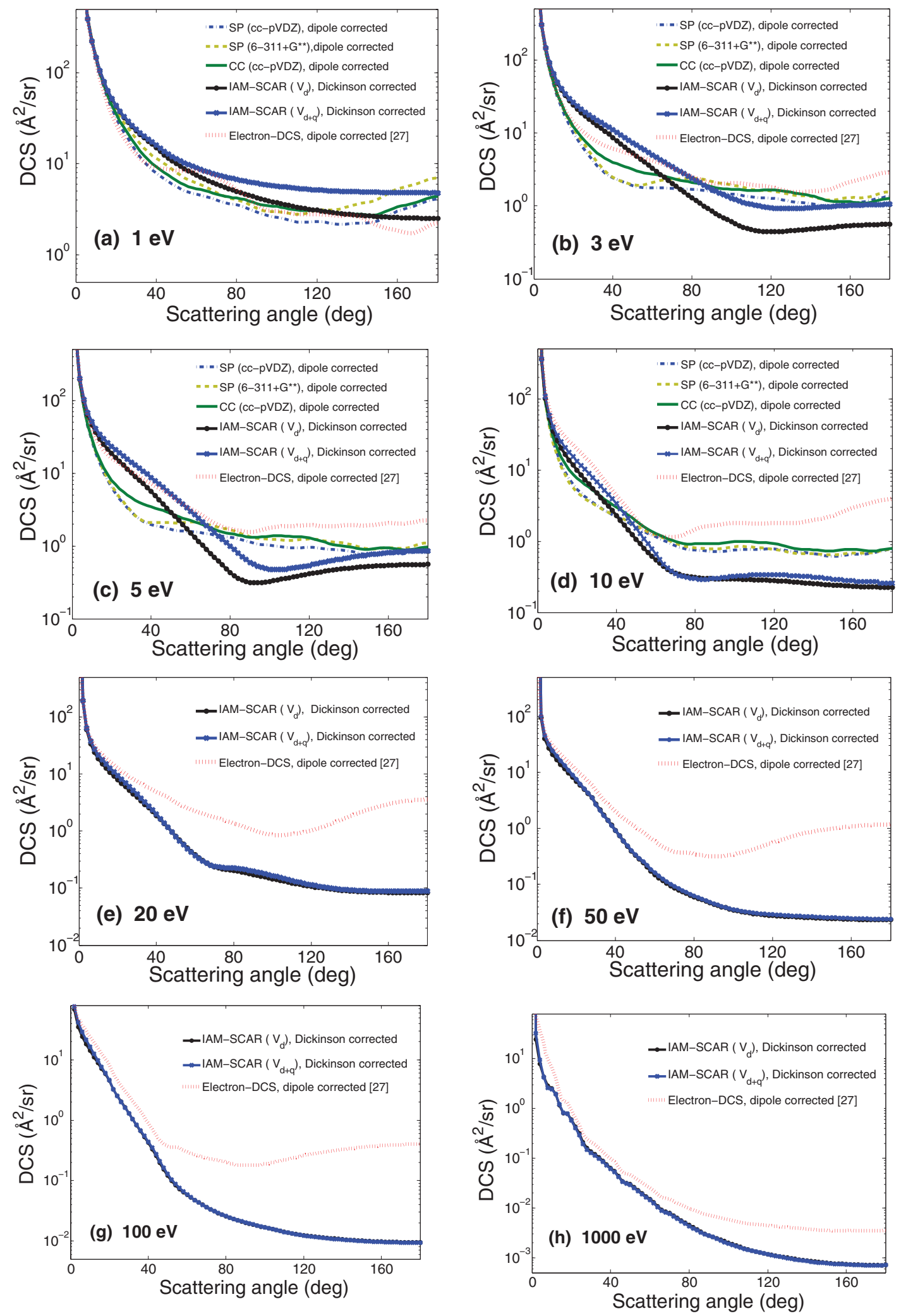

FIG. 3. (Color online) Elastic differential cross sections for positron scattering from pyrimidine, for the incident energies indicated in the panels. Results from the $R$-matrix approach at SP level using the cc-pVDZ (dashed-dotted blue line) basis and the diffuse basis set 6-311 + $\mathrm{G}^{* *}$ (dashed light-green line), and at the CC level with the compact basis set (solid dark-green line) are shown. Also plotted are the IAM-SCAR results with dipole polarization potential [solid black line $(\bullet)$ ] and dipole plus quadrupole polarization potential [solid blue line $(x)$ ]. All the $R$-matrix results are Born corrected and all the IAM-SCAR results are Dickinson corrected. For comparison we show the improved computed electron elastic DCS shown in Sanz et al. [27], as calculated with the $R$-matrix approach up to the ionization potential and then with IAM-SCAR method for higher energies. 


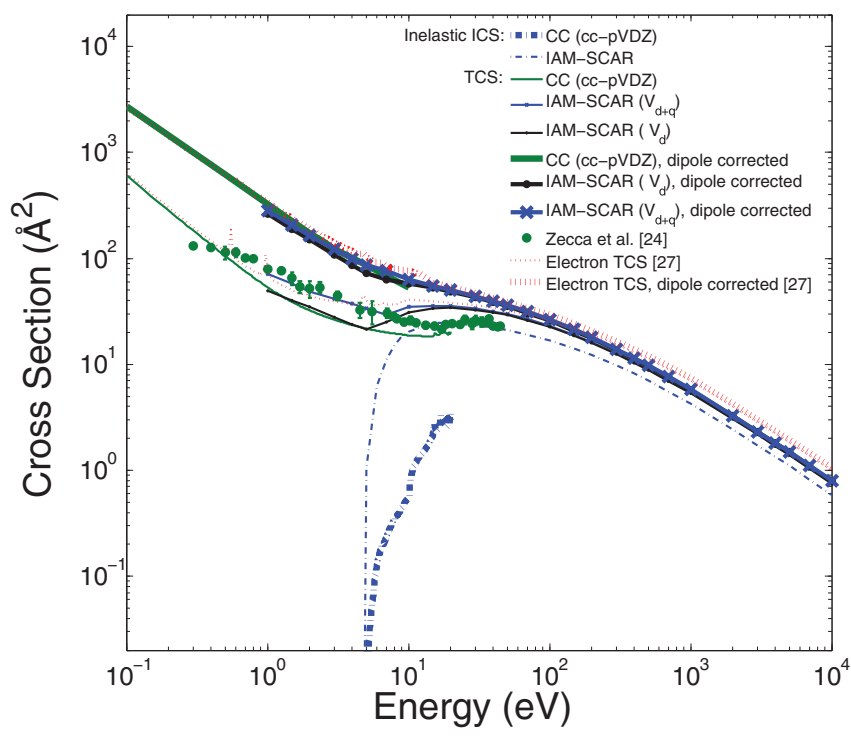

FIG. 4. (Color online) The present computed total cross sections for positron scattering by pyrimidine: $R$-matrix results at the CC level using the cc-pVDZ basis set (solid dark-green thin-line), IAM-SCAR results with dipole polarization potential [solid black thin line $(\bullet)$ ] and dipole plus quadrupole polarization potential [solid blue thin line $(x)$ ]. Also plotted are the TCS including the Born dipole-induced rotational excitations: $R$-matrix results at the CC level using the ccpVDZ basis set (solid dark-green thick line), IAM-SCAR results with dipole polarization potential [solid black thick line $(\bullet)$ ] and dipole plus polarization potential [solid blue thick line $(x)$ ]. These results are compared with the experimental data of Zecca et al. [24]. For comparison the computed electron total cross sections with (dotted red thick line) and without (dotted red thin line) Born correction, shown in Sanz et al. [27] are also plotted. In addition, the present inelastic integral cross sections computed with the $R$-Matrix approach (dashed-dotted blue thick line) and the IAM-SCAR (dashed-dotted blue thin line) method are given.

$\Delta j=1$ transition, which is the major contribution in particular at small angles. In Fig. 4 we also present total and inelastic ICSs computed with the IAM-SCAR approach, including the dipole and the dipole plus quadrupole polarization terms (see Table II). The inelastic ICS (dashed-dotted blue thin line) in this case comprises electronic excitations, ionization, and Ps formation, which were calculated through the atomic $i V_{a}(r)$ absorption potential and are therefore equal for both the $V_{d}$ and $V_{d+q}$ potentials (see Table II). Note that neither vibrations nor rotations are included in the IAM-SCAR inelastic integral cross sections. We observe an abrupt rise in the inelastic ICSs around $4.5 \mathrm{eV}$, that is, above both the Ps formation threshold and the first electronic-state excitation threshold. This means that any inelastic process lying below $4.5 \mathrm{eV}$ is at least in part ignored in the IAM-SCAR representation, indicating the limitations of the present independent-atom model in the lowenergy region. It is interesting to note that Mašín et al. [25] showed that the $R$-matrix electronically inelastic cross sections for electron collisions with pyrimidine tended to somewhat overestimate the experimental data; in contrast, for positron scattering they are well below the IAM-SCAR values. We attribute this discrepancy to the important role played by the Ps formation process at lower incident positron energies.
Regarding the total cross sections, we observe a significant rise in magnitude as the energy decreases. This low-energy tendency for positron-pyrimidine scattering, already observed in other polar species [81,82], is a consequence of the high permanent dipole moment and the strong polarizibility of this compound. This behavior is also observed for electron scattering [25] since it is independent of the sign of the charge of the projectile. In the low-energy domain, we see that the total $R$-matrix cross sections (solid dark-green thin line) are in fair agreement with the IAM-SCAR method [solid black thin line (•)] when using the $V_{d}$ potential (both uncorrected), since the polarization effects are described at the same level, while the $V_{d+q}$ cross sections [solid $\times$ blue thin line $(\times)$ ] are slightly larger.

Although good qualitative agreement is found, in terms of the energy dependence, between the present theoretical data and the experimental TCS data provided by Zecca et al. [24] (green dots), the computed TCSs (Born corrected as these account fully for the projectile-dipole interaction) are higher in magnitude than the measured TCSs. Nonetheless it is interesting to observe that the uncorrected IAM-SCAR TCSs, obtained with the dipole plus quadrupole polarization potential, shows good agreement with the measurements. This provides evidence in support of Chiari et al. [7] who claimed that the inclusion of the quadrupole polarizibility improves the results, in particular at low energies. Above the ionization threshold, both the $V_{d}$ and $V_{d+q}$ uncorrected IAM-SCAR results tend to converge although they overestimate to some extent the experimental values due to the increasing contribution of the inelastic channels. A similar situation was observed for positron- $\mathrm{O}_{2}$ scattering at intermediate energies, although for energies above $\sim 80 \mathrm{eV}$ the theory and experimental results converge [7]. Even though $e^{+}$-pyrimidine TCS were measured by Zecca et al. [24] only up to $50 \mathrm{eV}$, the trend of the last experimental values is to converge towards the theoretical curve. Recent experiments on positron scattering with the polar targets THF [82] and $3 \mathrm{H}$-THF [83] tend to diverge from the earlier measurements performed by Zecca et al. [84,85] at energies above Ps formation. Although no experimental explanation was given for these discrepancies, it may indicate that the TCSs from the Trento group are somehow underestimated in that energy regime.

We also observe in Fig. 4 good agreement between the Born-corrected $R$-matrix (solid dark-green thick line) and IAM-SCAR total cross sections [solid black thick line $(\bullet)$ and solid blue thick line $(\times)$ for $V_{d}$ and $V_{d+q}$, respectively], as mentioned above, due to the dominance of the dipole interaction. It is therefore somewhat paradoxical that the experimental TCS shows better agreement with the theoretical cross sections $\left(V_{d+q}\right.$ model) that neglect the dipole-induced rotational excitations. We believe this is mainly due to the angular discrimination of the experimental spectrometer, which provokes that the TCSs measured by Zecca et al. [24] miss part of the forward angle scattering contribution, such as the dipole-induced rotational excitations. Also, the limited energy resolution of the experiment implies that they are not able to distinguish rotationally excited molecules from the unscattered molecules. In a recent study Makochekanwa et al. [81] claimed that the forward effect leads to neglecting an 
important contribution to the cross section for polar molecules, in particular at low energies. For instance, the TCS measured for water at $0.5 \mathrm{eV}$ should be increased by around $\sim 67 \%$, and that for formic acid at $4 \mathrm{eV}$ by around $\sim 45 \%$ in order to account for this effect. Since the permanent dipole moment for pyrimidine is higher than for both those targets, we expect the forward angle scattering correction to be even more significant here. This effect may be responsible for the apparent disagreement between the measurements and theory [86], and therefore a realistic comparison can only be made between TCSs including rotational excitations and measured data corrected for the forward angle scattering effect.

It would be desirable to check this effect and compare our present positron-pyrimidine results with other theoretical or experimental data. There have been various attempts at studying positron scattering from the water molecule (see [6], [31]), which possesses a permanent dipole moment, although not as strong as pyrimidine $\left(\mu_{\mathrm{H}_{2} \mathrm{O}}=1.84 \mathrm{D}\right)$ [10]. For water, the experimental results differ by up to $50 \%$, in particular at low energies, due principally to the different acceptance angles in each experimental setup [6]. Moreover, the calculated cross sections [31] are considerably higher than the experimental values, since none of the three measurements is corrected for the forward angle scattering effect. Zhang et al. [6] provided a theoretical low-angle correction to the experimental cross sections of Beale et al. [87], thus improving the agreement between experiment and theory. It is probably legitimate to claim that a similar behavior is expected in the comparison between the present TCS results and the available measured data, with the apparent overestimation of our dipole-corrected theoretical data being due, at least in large part, to the lack of correction of the experimental data for forward angle scattering effects.

Finally, we have also included in Fig. 4 our electronpyrimidine TCS results [27]. The positron TCSs lie somewhat below the corresponding electron TCS, in particular at low energies. The most significant processes that distinguish positron and electron scattering, i.e., the exchange interaction and Ps formation, become small at energies above 100-200 eV. Despite the fact that the interaction probabilities for electron and positron scattering are therefore expected to converge at higher energies, we observe in Fig. 4 that the electron TCS remains slightly larger. These small discrepancies are not considered to be important, and can be attributed to the different atomic absorption potentials employed for positron and electron scattering [82].

\section{SUMMARY AND CONCLUSIONS}

In this study we have reported calculated elastic, inelastic, and total integral cross sections, together with the electronically elastic angular DCS, for positron scattering from the polar target pyrimidine over a very broad energy range from low energies $(0.1 \mathrm{eV})$ up to $10000 \mathrm{eV}$. Two different approaches were employed, depending on the collision energy range: the $R$-matrix method was used for energies up to $20 \mathrm{eV}$, although only data up to $10 \mathrm{eV}$ are listed in Table II, and a screening-corrected form of the independent-atom model (IAM-SCAR) was undertaken for intermediate to high energies, testing in each case the various models. Reasonable qualitative agreement is generally found between the different methods, but some discrepancies arise in particular at low energies. This agreement improves significantly once the cross sections are Born corrected to properly account for the effects of the large permanent dipole moment of pyrimidine (and therefore the dipole-induced rotational excitations): we found that our results were rather insensitive in this circumstance to the model employed as a consequence of the dominant role played by the dipole interaction over the static potential. This effect was also independent of the charge of the projectile, as was inferred from the similarity of the present positron cross sections to the analogous electron [27] scattering data. In addition, we noticed that the present Born-corrected TCSs were significantly bigger in magnitude than the experimental data of Zecca et al. [24]. We attributed this behavior to the fact that the angular discrimination of the experimental apparatus did not allow for the significant forward angle contribution (visible in the strongly forward peaked DCS) to be taken into account, providing underestimated TCS values. In addition, given that the experimental configuration did not have good enough energy resolution to distinguish rotationally excited molecules from unscattered ones, reasonable qualitative agreement arose between the measured TCS and the present computed TCS without a Born correction, in particular with the dipole plus quadrupole polarization IAM-SCAR model.

In conclusion, the present results showed that the combination of the $R$-matrix scattering method at low energies with the IAM-SCAR approach from intermediate to high energies provided a valid and realistic approach to study positron scattering over a very broad energy range, even if the target is a strong polar molecule. While perhaps not being strictly germane to the thrust of this paper, we also note the interesting review from Sadeghpour et al. [88], that in the near-threshold (low-energy) region provided analytic derivations to describe the magnitude and behavior of relevant cross sections in that energy regime. Nonetheless, ab initio results, such as we present here, are preferable if they are available. It is particularly interesting to have an evaluation of positron scattering cross sections with pyrimidine over a wide energy domain since these data are particularly relevant for radiation-based biomedical applications. Finally, we also believe that this scattering system would benefit from further measurements, at both the differential, integral, and total cross-section levels, and additional theoretical calculations.

\section{ACKNOWLEDGMENTS}

This work is partially supported by the Spanish Ministerio de Economía y Competitividad (Project No. FIS2012-31230) and the EU/ESF COST Action MP1002 "Nanoscale Insights into Ion Beam Cancer Therapy (Nano-IBCT)." M.C.F. has received a grant from the Comunidad Autónoma de Madrid local government. This work was also supported by EPSRC and the Australian Research Council through its Centres of Excellence programme. 
[1] S. Agostinelli et al., Nucl. Instrum. Methods Phys. Res., Sect. A 506, 250 (2003).

[2] J. Baró, J. Sempau, J. M. Fernández-Varea, and F. Salvat, Nucl. Instrum. Methods Phys. Res., Sect. B 100, 31 (1995).

[3] A. Muñoz, J. M. Perez, G. Garcia, and F. Blanco, Nucl. Instrum. Methods Phys. Res., Sect. A 536, 176 (2005).

[4] A. G. Sanz, M. C. Fuss, F. Blanco, F. Sebastianelli, F. A. Gianturco, and G. García, J. Chem. Phys. 137, 124103 (2012).

[5] M. C. Fuss, A. Muñoz, J. C. Oller, F. Blanco, M. J. HubinFranskin, D. Almeida, P. Limão-Vieira, and G. García, Chem. Phys. Lett. 486, 110 (2010).

[6] R. Zhang, A. Faure, and J. Tennyson, Phys. Scr. 80, 015301 (2009).

[7] L. Chiari, A. Zecca, S. Girardi, E. Trainotti, G. Garcia, F. Blanco, R. McEachran, and M. Brunger, J. Phys. B: At. Mol. Opt. Phys. 45, 215206 (2012).

[8] F. A. Gianturco, T. Mukherjee, and A. Occhigrossi, Phys. Rev. A 64, 032715 (2001).

[9] J. Franz, F. A. Gianturco, K. L. Baluja, J. Tennyson, R. Carey, R. Montuoro, R. R. Lucchese, T. Stoecklin, P. Nicholas, and T. L. Gibson, Nucl. Instrum. Methods Phys. Res., Sect. B 266, 425 (2008).

[10] CRC Handbook of Chemistry and Physics, edited by D. R. Lide (CRC, Boca Raton, FL, 1992-1993).

[11] I. Kulakowski, M. Geller, B. Lesyng, and K. L. Wierzcho, Biochim. Biophys. Acta. 361, 119 (1974).

[12] H. DeVoe and I. Tinoco, Jr., J. Mol. Biol. 4, 500 (1962).

[13] H. P. Weber and B. M. Craven, Acta Crystallogr. B. 46, 532 (1990).

[14] R. D. Nelson, Jr., D. R. Lide, and A. A. Maryott, Selected Values of Electric Dipole Moments for Molecules in the Gas Phase, NSRDS-NBS Series Vol. 10 (U.S. GPO, Washington, DC, 1967).

[15] G. L. Blackman, R. D. Brown, and F. R. Burden, J. Mol. Spectrosc. 35, 444 (1970).

[16] NIST Computational Chemistry Comparison and Benchmark Database, NIST Standard Reference Database No. 101, Release 15b, August 2011, edited by Russell D. Johnson III, http://cccbdb.nist.gov/

[17] J. B. Maljković, A. R. Milosavljević, F. Blanco, D. Šević, G. García, and B. P. Marinković, Phys. Rev. A 79, 052706 (2009).

[18] P. Palihawadana, J. P. Sullivan, M. J. Brunger, C. Winstead, V. McKoy, G. García, F. Blanco, and S. J. Buckman, Phys. Rev. A 84, 062702 (2011).

[19] D. B. Jones, S. M. Bellm, P. Limão-Vieira, and M. J. Brunger, Chem. Phys. Lett. 535, 30 (2012).

[20] D. B. Jones, S. M. Bellm, F. Blanco, M. C. Fuss, G. García, P. Limão-Vieira, and M. J. Brunger, J. Chem. Phys. 137, 074304 (2012).

[21] J. D. Builth-Williams, S. M. Bellm, D. B. Jones, H. Chaluvadi, D. H. Madison, C. G. Ning, B. Lohmann, and M. J. Brunger, J. Chem. Phys. 136, 024304 (2012).

[22] P. L. Levesque, M. Michaud, and L. Sanche, J. Chem. Phys. 122, 094701 (2005).

[23] F. Ferreira da Silva, D. Almeida, G. Martins, A. R. Milosavljević, B. P. Marinković, S. V. Hoffmann, N. J. Mason, Y. Nunes, G. García, and P. Limão-Vieira, Phys. Chem. Chem. Phys. 12, 6717 (2010).
[24] A. Zecca, L. Chiari, G. García, F. Blanco, E. Trainotti, and M. J. Brunger, J. Phys. B: At. Mol. Opt. Phys. 43, 215204 (2010).

[25] Z. Mašín, J. D. Gorfinkiel, D. B. Jones, S. M. Bellm, and M. J. Brunger, J. Chem. Phys. 136, 144310 (2012).

[26] J. R. Ferraz, A. S. dos Santos, G. L. C. de Souza, A. I. Zanelato, T. R. M. Alves, M.-T. Lee, L. M. Brescansin, R. R. Lucchese, and L. E. Machado, Phys. Rev. A 87, 032717 (2013).

[27] A. G. Sanz, M. C. Fuss, F. Blanco, Z. Mašín, J. D. Gorfinkiel, F. Carelli, F. Sebastianelli, F. A. Gianturco, and G. García, Appl. Radiat. Isot. (2013), doi: 10.1016/j.apradiso.2013.01.031.

[28] P. G. Burke, R-Matrix Theory of Atomic Collisions: Application to Atomic, Molecular and Optical Processes (Springer, Berlin, 2011).

[29] F. Blanco and G. Garcia, Phys. Lett. A 317, 458 (2003).

[30] F. Blanco and G. Garcia, Phys. Lett. A 330, 230 (2004).

[31] K. L. Baluja, R. Zhang, J. Franz, and J. Tennyson, J. Phys. B: At. Mol. Opt. Phys. 40, 3515 (2007).

[32] J. Tennyson, Phys. Rep. 491, 29 (2010).

[33] Z. Mašín and J. D. Gorfinkiel, J. Chem. Phys. 135, 144308 (2011).

[34] J. Tennyson, J. Phys. B: At. Mol. Phys. 19, 4255 (1986).

[35] G. Danby and J. Tennyson, J. Phys. B: At. Mol. Opt. Phys. 23, 1005 (1990).

[36] R. Zhang, K. L. Baluja, J. Franz, and J. Tennyson, J. Phys. B At. Mol. Opt. Phys. 44, 035203 (2011).

[37] J. M. Carr, P. Galiatsatos, J. D. Gorfinkiel, A. Harvey, M. Lysaght, D. Madden, Z. Mašín, M. Plummer, J. Tennyson, and H. Varambhia, Eur. Phys. J. D 66, 58 (2012).

[38] J. Franz, K. L. Baluja, R. Zhang, and J. Tennyson, Nucl. Instrum. Methods Phys. Res., Sect. B 266, 419 (2008).

[39] N. F. Lane, Rev. Mod. Phys, 52, 29 (1980).

[40] D. W. Norcross and L. A. Collins, Adv. Atom. Mol. Phys. 18, 341 (1982).

[41] M. A. Morrison, Adv. At. Mol. Phys. 24, 51 (1988).

[42] D. W. Norcross and N. T. Padial, Phys. Rev. A 25, 226 (1982).

[43] F. A. Gianturco and A. Jain, Phys. Rep. 143, 347 (1986).

[44] O. H. Crawford, J. Chem. Phys. 47, 1100 (1967).

[45] N. Sanna and F. A. Gianturco, Comput. Phys. Commun. 114, 142 (1998).

[46] I. Rabadán and J. Tennyson, Comput. Phys. Commun. 114, 129 (1998).

[47] Y. Okamoto, K. Onda, and Y. Itikawa, J. Phys. B 26, 745 (1993).

[48] F. Blanco and G. Garcia, Phys. Lett. A 295, 178 (2002).

[49] F. Blanco and G. García, Phys. Rev. A 67, 022701 (2003).

[50] R. P. McEachran, J. P. Sullivan, S. J. Buckman, M. J. Brunger, M. C. Fuss, A. Muñoz, F. Blanco, R. D. White, Z. Lj. Petrović, P. Limão-Vieira, and G. García, J. Phys. B 45, 045207 (2012).

[51] D. D. Reid and J. M. Wadehra, Phys. Rev. A 50, 4859 (1994).

[52] D. D. Reid and J. M. Wadehra, J. Phys. B: At. Mol. Opt. Phys. 29, L127 (1996).

[53] D. D. Reid and J. M. Wadehra, J. Phys. B: At. Mol. Opt. Phys. 30, 2318 (1997).

[54] X. Z. Zhang, J. F. Sun, and Y. F. Liu, J. Phys. B 25, 1893 (1992).

[55] R. P. McEachran, D. L. Morgan, A. G. Ryman, and A. D. Stauffer, J. Phys. B 10, 663 (1977).

[56] T. M. Miller and B. Bederson, Adv. At. Mol. Phys. 13, 1 (1978).

[57] A. K. Bhatia, Positron Interactions with Atoms and Ions (NASA Goddard Space Flight Center, Greenbelt, MD, 2012).

[58] R. D. Alpher and D. R. White, Phys. Fluids 2, 153 (1959).

[59] E. A. Reinsch and W. Meyer, Phys. Rev. A 18, 1793 (1978). 
[60] H. J. Werner and W. Meyer, Phys. Rev. A 13, 13 (1976).

[61] A. C. L. Jones, C. Makochekanwa, P. Caradonna, D. S. Slaughter, J. R. Machachek, R. P. McEachran, J. P. Sullivan, S. J. Buckman, A. D. Stauffer, I. Bray, and D. V. Fursa, Phys. Rev. A 83, 032701 (2011).

[62] D. J. R. Mimnagh, R. P. McEachran, and A. D. Stauffer, J. Phys. B: At. Mol. Opt. Phys. 261727 (1993).

[63] G. Staszewska, D. W. Schwenke, D. Thirumalai, and D. G. Truhlar, Phys. Rev. A 28, 2740 (1983).

[64] N. Hush and A. S. Cheung, Chem. Phys. Lett. 34, 11 (1975).

[65] M. Charlton and J. W. Humberston, Positron Physics (Cambridge University Press, Cambridge, 2001).

[66] J. W. Otvos and D. P. Stevenson, J. Am. Chem. Soc. 78, 546 (1956).

[67] Y. Jiang, J. Sun, and L. Wan, Phys. Rev. A 52, 398 (1995).

[68] P. Limão-Vieira, F. Blanco, J. C. Oller, A. Muñoz, J. M. Pérez, M. Vinodkumar, G. García, and N. J. Mason, Phys. Rev. A 71, 032720 (2005).

[69] P. Limão-Vieira, M. Horie, H. Kato, M. Hoshino, F. Blanco, G. Garcia, S. J. Buckman, and H. Tanaka, J. Chem. Phys. 135, 234309 (2011).

[70] H. Kato, A. Suga, M. Hoshino, F. Blanco, G. García, P. Limão-Vieira, M. J. Brunger, and H. Tanaka, J. Chem. Phys. 136, 134313 (2012).

[71] A. Jain, J. Phys. B 21, 905 (1988).

[72] A. Muñoz, J. C. Oller, F. Blanco, J. D. Gorfinkiel, P. LimãoVieira, and G. García, Phys. Rev. A 76, 052707 (2007).

[73] A. Zecca, L. Chiari, G. García, F. Blanco, E. Trainotti, and M. J. Brunger, New J. Phys. 13, 063019 (2011).

[74] A. S. Dickinson, J. Phys. B 10, 967 (1977).

[75] M. Schreiber, M. R. Silva-Junior, S. P. A. Sauer, and W. Thiel, J. Chem. Phys. 128, 134110 (2008).
[76] A. Faure, J. D. Gorfinkiel, L. A. Morgan, and J. Tennyson, Comput. Phys. Commun. 144, 224 (2002).

[77] H. J. Werner, P. J. Knowles, R. Lindh, F. R. Manby, M. Schütz et al., MOLPRO, version 2009.1, a package of ab initio programs, 2009, see http://www.molpro.net.

[78] G. Fischer, Z. L. Cai, J. R. Reimers, and P. Wormell, J. Phys. Chem. A 107, 3093 (2003).

[79] Z. Mašín, Ph.D. thesis, The Open University, 2012.

[80] S.-I. Chu and A. Dalgarno, Phys. Rev. A 10, 788 (1974).

[81] C. Makochekanwa, A. Banković, W. Tattersall, A. Jones, P. Caradonna, D. S. Slaughter, K. Nixon, M. J. Brunger, Z. Petrović, J. P. Sullivan, and S. J. Buckman, New J. Phys. 11, 103036 (2009).

[82] L. Chiari, E. Anderson, W. Tattersall, J. R. Machacek, P. Palihawadana, C. Makochekanwa, J. P. Sullivan, G. García, F. Blanco, R. P. McEachran, M. J. Brunger, and S. J. Buckman, J. Chem. Phys. 138, 074301 (2013).

[83] L. Chiari, P. Palihawadana,, J. R. Machacek, C. Makochekanwa, G. García, F. Blanco, R. P. McEachran, M. J. Brunger, S. J. Buckman, and J. P. Sullivan, J. Chem. Phys. 138, 074302 (2013).

[84] A. Zecca, C. Perazzolli, and M. J. Brunger, J. Phys. B 38, 2079 (2005).

[85] A. Zecca, L. Chiari, A. Sarkar, and M. J. Brunger, J. Phys. B: At. Mol. Opt. Phys. 41, 085201 (2008).

[86] J. P. Sullivan, C. Makochekanwa, A. Jones, P. Caradonna, D. S. Slaughter, J. Machacek, R. P. McEachran, D. W. Mueller, and S. J. Buckman, J. Phys. B: At. Mol. Opt. Phys. 44, 035201 (2011).

[87] J. Beale, S. Armitage, and G. Laricchia, J. Phys. B: At. Mol. Opt. Phys. 39, 1337 (2006).

[88] H. R. Sadeghpour, J. L. Bohn, M. J. Cavagnero, B. D. Esryk, I. I. Fabrikant, J. H. Macek, and A. R. P. Rau, J. Phys. B 33, R93 (2000). 